\title{
COMENTARIO
}

\section{Mahan y la geopolítica}

\author{
Andrés GONZÁLEZ MARTÍN \\ Escuela Superior de las Fuerzas Armadas \\ Centro Superior de Estudios de la Defensa Nacional \\ Federico AZNAR FERNÁNDEZ-MONTESINOS \\ Instituto Español de Estudios Estratégicos \\ Centro Superior de Estudios de la Defensa Nacional \\ faznfer@fn.mde.es
}

\section{REFERENCIA NORMALIZADA}

González Martín, Andrés, y Aznar Fernández-Montesinos, Federico (2013) "Mahan y la geopolítica". Geopolítica(s). Revista de estudios sobre espacio y poder, vol. 4, núm. 2, 335-351.

\section{Mahan marino de guerra}

El Contralmirante Alfred Thayer Mahan obtuvo su último empleo militar en 1906, diez años después de retirarse del servicio activo y como consecuencia de un decreto que otorgaba el ascenso a todos los Capitanes de Navío que hubiesen participado en la Guerra Civil Americana. Su ascenso a Almirante fue un ascenso honorifico en bloque a un grupo de viejos oficiales. No fue, por lo tanto, un ascenso por su meritoria carrera como marino de guerra.

Y es que Mahan no fue un gran marino de Guerra. Tampoco era un entusiasta de las nuevas tecnologías. No le gustaban los humeantes barcos de vapor y parecía soñar con la vuelta a la navegación de vela. Incluso Philip. A Crowl (1992), en el famoso libro dirigido por Peter Paret, "Creadores de la Estrategia Moderna", deja caer que intentó evitar los destinos en el mar. De hecho, pasó a la historia no por lo que hizo en el mar sino por los libros y artículos que escribió. Este tipo de casos no son muy frecuentes pero en algunas ocasiones aparecen soldados que con sus palabras dejan una huella profunda y duradera. Entre este selecto grupo de militares podemos encontrar al General Karl Von Clausewitz, el más famoso filósofo de la guerra de Occidente, al General Antoine Henri de Jomini, el teórico de la guerra más destacado del siglo XIX, al Capitán del Ejército Británico Basil Henry Liddell Hart, padre de la Estrategia de la aproximación indirecta, al General John Frederick 
Charles Fuller, creador de la doctrina de guerra acorazada, al General Karl Haushofer, catedrático de Geografía en Múnich, famoso geopolítico y precursor del concepto de Lebensraum, y por supuesto el Capitán de Navío y Contraalmirante honorifico Alfred Thayer Mahan.

Un hombre clave en su vida profesional fue el Almirante Stephen Luce, primer director del Naval War College quien pensó en Mahan, como uno de sus posibles profesores, porque había leído un libro suyo sobre la historia naval de la Guerra de Secesión Norteamericana, titulado El Golfo y las aguas interiores (Mahan, 1883).

Por otra parte, Alfred Thayer Mahan no pertenecía a una familia de tradición naval. Su padre fue un oficial del Ejército de Tierra, Dennis Hart Mahan, número uno de su promoción en West Point; la mayoría de sus años de servicio los pasó como profesor, escritor e investigador en West Point, donde precisamente nació el gran teórico del poder naval. A su posteriormente famoso hijo decidió ponerle el nombre de Thayer, en honor del General de Ejército de Tierra Silvanus Thayer, reconocido como "the Father of West Point" y su fundador. En la actualidad en la Academia militar de West Point hay una sala dedicada a Mahan pero no al hijo marino sino al señor Dennis Hart, el padre y oficial del ejército. Por tanto, el tridente norteamericano de Neptuno era hijo de un oficial del Ejército de Tierra que enseñaba matemática, ingeniería y estrategia a los cadetes del Army. Además nació en West Point, la Academia del Ejército de Tierra, llevaba el nombre del General fundador de la Academia General Militar de los Estados Unidos y su pericia en el mar estaba puesta en cuestión.

\section{Mahan historiador}

El libro más importante de Mahan (1890), La influencia del poder naval en la historia 1660-1783, no es, ni mucho menos, un trabajo de investigación —no pretende en ningún momento ser objetivo y no lo disimula - sino un documento político que se sirve de la Historia de un modo reduccionista con vistas a justificar una concreta propuesta política; ésta no es el punto de partida de la investigación por más que se presente como tal, sino más bien sus conclusiones.

Mahan es un oficial naval, su formación es por tanto técnico-científica, aunque coyunturalmente actúe como docente; comienza a publicar con 50 años de edad en un ámbito para el que no ha recibido una formación específica, lo que permite vaticinar en su trabajo importantes carencias conceptuales, pero también aporta una nueva aproximación, reflexiones realizadas desde otros ámbitos, aire fresco.

Uno de sus principales valores es la intuición. Compone su discurso a partir de elementos estratégicos, operacionales e incluso tácticos de los que extrae consecuencias políticas. Para eso emplea el código binario - bien-mal, todo-nada - de los acontecimientos, un lenguaje extraordinariamente poderoso y sugerente como todo reduccionismo, y lo emplea para analizar los supuestos, obviando de modo 
partidista y sin más ni mayor explicación aquellos otros que no encajen con sus ideas o le contradigan, lo cual lo hace muy didáctico.

Su utilización de la Historia, y más aún en el caso español, es interesada porque el eje de su propuesta pasa por hacerse con los restos de su imperio colonial y su descrédito era un paso adelante en su apuesta política, una cuestión en la que la voluntad se presenta como capital al generar un espacio para la ilusión y el futuro. Además, y como se verá, su propuesta es el modelo de imperio inglés en cuyo mito fundacional está la lucha contra España.

Mahan básicamente recogía en su trabajo la práctica política de su país. Los planes de construcción naval se iniciaron en la década de 1880; en 1873 apareció la prestigiosa revista de temas navales Proceedings y en 1884 se creó la Escuela de Guerra Naval. En ese momento, el más oportuno, apareció Mahan que no sólo recogía el pensamiento de los líderes navales norteamericanos y sus lugares comunes, sino que añadía sus propias aportaciones, sistematizándolo y extrayendo conclusiones que sirven para señalizar sus siguientes y necesarios pasos cuya justificación es precisamente el objeto del trabajo. A su vez, su obra sirvió para publicitar el esfuerzo naval norteamericano, estimularlo y reconocer su valor y utilidad.

Su propuesta política enlaza la "Doctrina Monroe" con el Big Stick del presidente Theodore Roosevelt perfeccionando la doctrina del "Destino Manifiesto", originalmente orientada hacia las Américas a la que convierte en una formulación para el mundo. En ella el Poder Naval asume las funciones de policía que proclama su compromiso con la difusión de la democracia. Además, se aproximó al concepto de interés nacional para la articulación de la política exterior del país y fue el autor de términos ampliamente difundidos como "Oriente Medio".

A la vista de la trayectoria de Mahan se puede aseverar que si no fue un brillante marino tampoco fue un brillante historiador. Realmente es dudoso que le importase demasiado la historia, aunque eso no quiere decir que no se sintiese atraído por la idea de ser protagonista de la historiografía de la nueva potencia americana. El interés del Contralmirante estaba dirigido más a la elaboración del pasado que al propio pasado, colaborando en la producción de una imagen que se utilizará a favor de ciertos objetivos no relacionados con la investigación histórica. Mahan en sus libros pretende demostrar que el éxito del Imperio británico tiene su origen en su capacidad de dominar las olas. Nadie duda de la relevancia del factor naval en la construcción de poder nacional británico, pero Mahan confunde condición necesaria con condición suficiente. Si Nelson fue un héroe con su victoria en Trafalgar no es menos cierto que Sir Arthur Wellesley, el Duque de Wellington, lo es también con su victoria en Waterloo. Si John Churchill, Duque de Marlborough, venció brillantemente en el mar, estas hazañas desde luego no fueron tan destacadas como las que obtuvo en tierra durante la guerra de sucesión española a principio del siglo XVIII. Si Winston Churchill fue Lord del Almirantazgo, también es verdad que fue Teniente de Caballería y participo en la lucha contra las revoltosas tribus pastunes en la frontera afgano pakistaní y en la campaña de Sudán, tomando parte en la batalla 
de Omdurmán, donde la caballería británica lanzó una de sus últimas cargas. La delgada línea roja de casacas británicas, su diplomacia, el poder de su mercado financiero, el éxito de su revolución industrial y técnica no pueden dejarse a un lado como factores de peso en la construcción de la paz británica de su Imperio. No en vano, cuando el general norteamericano George C. Marshall, pasó a ejercer como Secretario de Estado (Exteriores), los periodistas le preguntaron cómo se sentía ante un cambio tan radical. Su respuesta es legendaria: "En ambos cargos hago lo mismo: administro los límites del poder" (cit. en Zorrilla, 2013).

Mahan en su relación con la historia nunca buscó ser objetivo, ni metódico, ni riguroso. Tampoco buscó la precisión de una investigación completa de un asunto. En 1902 siendo presidente de la American Historical Association, afirmaba que la historia escrita consistía en un conjunto de detalles subordinados alrededor de una idea central. Para Mahan algunos sucesos no merecían la pena. Para él los hechos hay siempre que subordinarlos al tema central. Lo importante es la idea que situamos en el centro de gravedad, lo demás sólo sirve para vestir su difusión. Esta forma de tratar al pasado no es la que se enseña en las facultades de Historia. La falta de rigor académico a la hora de tratar los acontecimientos históricos puede ser un pecado en el mundo de los profesores y de los intelectuales pero no necesariamente en el entorno del poder y en el ámbito estratégico. El profesor Heriberto Cairo selecciona una interesante definición de Yves Lacoste:

La Geografía es, en primer lugar, un saber estratégico estrechamente unido a un conjunto de prácticas políticas y militares, y son dichas prácticas las que exigen la recopilación articulada de unas informaciones extremadamente variadas y a primera vista heterogéneas, cuya razón de ser y cuya importancia no es posible entender si nos limitamos a la legitimidad del Saber por el Saber (Cairo, 2005: x).

De ahí que se produjese, según Lacoste, un divorcio entre la Geografía de los profesores y la de los Estados Mayores. Con la historia pasa lo mismo. La Geografía, la Geografía política, la Geopolítica y la Historia, aunque no solo ellas, son para los militares y políticos también disciplinas estratégicas, es decir orientadas a la acción no a la disquisición.

La influencia del poder naval en la historia 1660-1783 es un libro que pretende ser de historia militar, su autor así lo dice en la primera página de la introducción del libro:

La historia del poder naval, a la vez abarca en un ancho campo todo lo que tiende a hacer a un pueblo grande en el mar y por el mar, es en gran parte una historia militar, y bajo este aspecto la mostramos principalmente en este libro, aunque no de un modo exhaustivo (Mahan, 1901: 2). 
No se puede acusar al autor de no ser sincero. Reconoce desde el principio que no será exhaustivo.

\section{Mahan profesor militar}

Mahan como profesor de Historia Naval no sigue el típico patrón de profesor militar de Historia; él es un profesor de Estado Mayor y el sentido de su enseñanza es muy otro. Tiene que estimular el espíritu crítico, la participación activa del alumno y convertirlo en el centro del proceso de aprendizaje. Debe romper moldes, clichés y ataduras. Estamos en una fase del ciclo formativo del Oficial, ya en el ecuador de su carrera, en que éste deja de mirar al suelo y aprende a mirar al cielo, a tener apertura de mente, a hilvanar ideas y a componer un discurso completo. Si no lo logra su carrera se resentirá.

Podemos estar o no de acuerdo con sus aportaciones, con sus convicciones y con su metodología pero lo que no podemos negar es que Mahan tenía ideas propias, que supo adaptar a un mecanismo usualmente inútil y transmitirlas no solo a sus alumnos, sino también al más alto nivel de decisión política. A Mahan no le importaba mucho que sus alumnos fueran capaces de memorizar con precisión los más nimios detalles de la batalla de Trafalgar. Lo que le parecía sin embargo trascendental es que supieran por qué el Reino Unido ganó la guerra y los demás la perdieron. Para Mahan, la historia militar es algo más que batallas y esto desde luego es una consideración excepcional en la enseñanza militar de esta materia. Pero siendo esto verdad se vale también de las batallas. Habla en los términos que se espera que hable para fijar algunas ideas que se repiten como un estribillo continuamente a lo largo de los ejemplos históricos que elige. El resultado final no puede ser otro que el adoctrinamiento ilustrado, que no es menos peligroso que el adoctrinamiento a secas pero que seguramente tiene algo más de gracia.

Las clases de historia de Mahan no tienen solo la función de descubrir lo que pasó sino que fundamentalmente tienen una función pedagógica, en tanto que permiten pulir la sensibilidad de los nuevos mandos de la Armada de los Estados Unidos. Crowl nos recuerda un comentario del Secretario de Guerra, bajo la presidencia de Franklin Roosevelt, Henry Stimson:

La peculiar psicología del Departamento de la Marina de los Estados Unidos a menudo parecía abandonar el reino de la lógica para introducirse en un confuso mundo religioso, en el que Neptuno era su dios, Mahan su profeta y la US Navy la única iglesia verdadera (Crowl, 1992: 483).

Para la marina de los Estados Unidos su profeta articula un discurso sin fisuras que justifica su relevante papel en la historia de la nación elegida y por lo tanto del mundo. La historia militar es sólo el soporte discursivo sobre el que diseñar una 
completa reconceptualización de la proyección del poder nacional de los Estados Unidos, tarea que tiene efectos inmediatos en el diseño de una estrategia genética de fuerza que permitió a los Estados Unidos, a final del siglo XIX, convertirse en una potencia naval en el mundo, aspiración que nunca había formado parte del proyecto nacional de los padres fundadores.

\section{Mahan propagandista}

En general, los militares - aunque no solo ellos, ya que esta postura es muy común en la formación técnica- prefieren ver y tocar a oír y gustar. Las presentaciones militares suelen ser muy brillantes y convincentes, se han acompañado siempre de mapas, colores, símbolos de todo tipo para representar toda clase de unidades, flechas rojas o azules, punteros, dibujos, fotografías, películas y ahora con las nuevas posibilidades de la informática y del Power Point mucho más. A los militares les encantan los cajones de arena, los bancos de pruebas y la simulación porque es la forma de reducir la realidad a modelos más simples que resultan manejables y, por tanto, operativos, útiles.

El elemento de referencia del Oficial por antonomasia no es lo estratégico -éste queda reservado para el General o incluso para el político; y además se muestra demasiado inconcreto- sino el operativo, tangible, claro; el buen Oficial es "operativo", decide, resuelve. Tal es su formación: su tendencia natural es hacia lo concreto, lo útil, y en el plano temporal hacia lo crematístico, el cortoplacismo que siempre lleva asociado el riesgo de lo epidérmico. Le cuesta trabajo escapar de ese ciclo, pero si quiere prosperar profesionalmente debería hacerlo, por más que siempre le quede el poso de lo operativo; es más, siempre trata de volver a este tiempo, donde el mundo era ordenado y comprensible, y él, joven como Peter Pan, disfrutaba de su felicidad primera.

La imaginación es peligrosa al menos de Comandante / Capitán de Corbeta para abajo. Pero si la disciplina es la norma, a la hora de mandar, de Almirante / General a Comandante - y más conforme mayor es el grado - hay que exigirle algo más que cumplir órdenes y leer reglamentos militares. La responsabilidad del General exige libertad para decir que no a las exigencias de una doctrina o de unas ordenes si su conciencia o su razón le animan a ello. Esta libertad exige criterio, capacidad de reconsiderar lo que viene de arriba y valor moral para cuestionarlo si lo considera oportuno.

Federico el Grande decía "conocimientos puede tenerlos cualquiera, pero el arte de pensar es el regalo más escaso de la naturaleza", y esta diferencia importante es el eje de los Altos Estudios Militares. Da la impresión que el conocimiento de las cosas tiene que ver más con ver y tocar y que pensar se relaciona en mayor medida con el oír y gustar, dando por supuesto que lo que se oye y se gusta no siempre es 
agradable. Se conoce focalizando la atención y se piensa oyendo otras voces que se contrastan con la originalmente propia. Quevedo lo habría dicho en verso:

Retirado en la paz de estos desiertos, / con pocos, pero doctos libros juntos,/ vivo en conversación con los difuntos/ y escucho con mis ojos a los muertos./ Si no siempre entendidos, siempre abiertos,/o enmiendan, o fecundan mis asuntos;/ y en músicos callados contrapuntos/al sueño de la vida hablan despiertos (cit. en Fanconi, 1995).

Mahan en su libro La influencia del poder naval en la historia escribió mucho, dijo muchas cosas, pero transmitió sólo unas cuantas ideas, eso sí muy sugestivas, especialmente para un marino o para un amigo de los grandes garrotes como Theodore Roosevelt: "Habla con suavidad y lleva un buen garrote; llegarás lejos".

Pocas ideas-fuerza repetidas y sin matices son desde luego algo parecido a la propaganda, y la propaganda es el más fuerte instrumento político si se emplea con oportunidad. Esto no quiere decir que Mahan fuera un oportunista pero lo que sí es verdad es que su libro fue muy oportuno. Los Estados Unidos de América precisamente en 1890 reconocen el cierre de la frontera terrestre sin colonizar (Jiménez, 2001). La frontera se había esfumado repentinamente y los norteamericanos ya sólo tenían ante sí agua salada y las naciones del más allá. Por primera vez la expansión ya solo era posible a través del mar y no por tierra. El espíritu de frontera que descubriera Frederick Jackson Turner (1893) estaba en peligro. Desde la óptica de Turner, para quien la frontera había desempeñado un papel crucial en la creación de la identidad de las instituciones y la nación norteamericana, la noticia del fin de la frontera era el fin de una misión, pero seguramente no el de un destino. Delante ya no había tierra libre desocupada pero si agua libre sin dueño. El sueño americano del "destino manifiesto", al que John O'Sullivan, editor de la Democratic Review, dio vida en 1845 al llegar al Pacífico, se reconfiguró y Mahan abrió la puerta de un nuevo escenario sin explorar por los norteamericanos, donde la Providencia pudiera seguir actuando a favor del impulso civilizador de los Estados Unidos.

Las pequeñas trece colonias habían ocupado todo el continente hasta llegar a la costa del Pacífico y en ese momento, cuando el espacio vital americano, el particular y al parecer democrático Lebensraum de la república constitucional del "We the People", estaba dominado, surge una visión del mundo, del poder y su función renovadora. Mahan se encarga de dar sentido a una aspiración que antes no había mostrado su pulso. Hablar de Lebensraum norteamericano no es una ocurrencia, el mismo Adolfo Hitler, cuando empezó a pensar en poner en marcha la operación Barbarroja, se refería al Este de Europa como el "Far West" alemán. Si esas pequeñas colonias habían sido capaces de construir un gran sueño americano conquistando para la civilización de manos de pueblos inferiores todo un continente, Alemania no sería menos. Ian Kershaw (2010), en su prestigiosa biografía de Adolfo Hitler, pone en boca del dictador nazi en varias ocasiones la analogía que el nacional 
socialismo y especialmente su Führer veían entre el oeste americano y el este europeo. Uno y otro territorio constituían el espacio vital necesario para que una raza pudiera arraigar y sobrevivir con la dignidad de su condición superior. Por cierto, y también lo cita Kershaw, a Hitler le encantaban las películas y novelas de vaqueros, es decir las del Oeste.

La mitificación de la conquista del Oeste y la nueva frontera de Turner inspiró sin duda el sueño expansionista de Hitler. Si los norteamericanos habían mitificado el fenómeno histórico de la ocupación de millones de kilómetros cuadrados sin ocuparse de una interpretación objetiva de la realidad anterior, el movimiento de masas Nacional Socialista, con un genio de la propaganda como Goebbels, no tendría ningún tipo de problema en justificar la germanización de los territorios ocupados a los pueblos eslavos inferiores. Si en los Estados Unidos la presión social y política había elaborado una ficción épica de la conquista del Oeste, Alemania haría lo mismo.

Desgraciadamente para España nos tocó estar en medio de las nuevas ambiciones norteamericanas en el mar. Cuba, Puerto Rico, Filipinas y Guam en el archipiélago de las Marianas son pruebas del éxito de la nueva doctrina del poder naval para una nueva potencia, que toma conciencia de su poder nacional gracias al destacado propagandista Mahan.

\section{Mahan geopolítico}

Es norma común en las actividades humanas que la doctrina vaya por detrás de los acontecimientos que han servido a su formulación. Cuando aparece la palabra con la que se sintetiza la fórmula, ésta es objeto de una suerte de reverencia, como si del inicio de un nuevo tiempo se tratara y nunca antes se hubiera hecho o existido tal cosa. Esto también sucede con la geopolítica, una palabra que desde una perspectiva no académica, encierra múltiples significados y que durante un tiempo fue usada con profusión y a la que se asignaba el rigor y la exactitud científica, para luego proscribirla como causa cierta de toda una guerra mundial. Así la naturaleza pendular del proceder humano, de sus intuiciones transformadas en dogmática claridad de ideas, determina que después de Kjellen, que en 1899 formulara el concepto, todo fuera geopolítica -interpretada, además, en clave determinista- y tras 1945, cuando se declaró a la geopolítica culpable, nada.

El pensamiento de Mahan se desarrolla a la par que el surgimiento de la geopolítica, entre 1890 - cuando publicó su obra cumbre, La influencia del poder naval en la Historia, construida sobre sus lecciones como profesor en la Escuela de Guerra Naval-y 1911. De hecho, se puede decir que Mahan habla en clave geopolítica sin identificarla como tal, al menos al principio.

La propuesta de Mahan se inscribe en lo que Agnew (2005) clasificaba como la era de la geopolítica naturalizadora propia de la rivalidad política interimperial que 
se dio en el período comprendido entre 1875 y 1945 . Ésta divide el mundo entre pueblos imperiales y colonizados, al tiempo que afirma que los Estados tienen "necesidades biológicas" de territorio y recursos así como de dar salidas a sus empresas. El mundo se encuentra cerrado y plantea la situación mundial como un juego de suma cero. Aún es más, el mundo tiene características geográficas y condiciones medioambientales estables con efectos predecibles en el status global de los Estados; son los imperativos de la geografía de los que no resulta posible escapar.

Y es que la aplicación de concepciones darwinistas en el ámbito de las Relaciones Internacionales se traducirán en una competencia por las colonias. En palabras de Bagehot, "la naturaleza es roja en diente y garra", y "en cualquier situación del mundo, las naciones más fuertes tienden a prevalecer sobre las otras; y en ciertas peculiaridades especiales, los más fuertes tienden a ser los mejores" (cit. en Brodie, 1978: 256).

En esta línea, Lord Salisbury en 1898, y en el contexto de un célebre discurso de gran trascendencia para España, aseveraba:

Podemos dividir las naciones del mundo en vivas y moribundas. Por un lado, tenemos naciones cuyo poder aumenta cada año, aumentando su riqueza, aumentando su poder, aumentando la perfección de su organización. Los ferrocarriles le han dado el poder de concentrar en un solo punto la totalidad de la fuerza militar de su población [...] La ciencia ha colocado en manos de esos Ejércitos armamentos que aumentan cada vez más su fuerza destructiva [...] Junto a estas esplendidas organizaciones...que sostienen ambiciones encontradas que únicamente el futuro podrá dirimir a través de un arbitraje sangriento [...] Existen un número de comunidades que sólo puedo describir como moribundas [...] son comunidades principalmente no cristianas, aunque siento decir que no es éste exclusivamente el caso, y en esos Estados, la desorganización y la decadencia avanzan casi con tanta rapidez como la concentración y aumento de poder en las naciones vivas que se encuentran junto a ellos. Década tras década cada vez más débiles, más pobres y poseen menos hombres destacados o instituciones en que poder confiar [...] [Los medios de comunicación] las describen con los más conspicuos tintes apelando tanto a sus sentimientos como a sus intereses, pidiendo que se les ponga remedio [...] por una u otra razón, por necesidades políticas o bajo presiones filantrópicas, las naciones vivas se irán adueñando gradualmente de los territorios de las moribundas y surgirán rápidamente las semillas y las causas de conflicto entre las naciones civilizadas [...] [Pero] no sentiremos envidia si el engrandecimiento de un rival elimina la desolación y la esterilidad de regiones en las que nuestros brazos no pueden alargarse (cit. en Pérez Tudela Baez, 1992: 73).

Mahan veía en la guerra una "ley de progreso", como también lo hacían pensadores como el propio Renan. La guerra, para él, es simplemente un movimiento político, aunque violento y excepcional en su carácter. Y aun es más, apuesta por su uso para la cristianización del mundo. Rudolf Kjellen (1916), acepta las tesis darwinistas y también considera al Estado un organismo vivo sometido a todos los 
avatares biológicos. Esta dinámica de afianzamiento y expansión es, a su juicio, la base de la geopolítica. Este mismo enfoque daría paso a construcciones como la del concepto de Lebensraum, elaborado por Ratzel, desarrollo geopolítico con el que se señala el espacio vital necesario para el desarrollo de un pueblo, y que posteriormente Haushoffer aplicara a la Alemania de después de Versalles. Ratzel, en un discurso que recuerda al "Diálogo de Melos" (Tucídides, 1990), decía:

La lucha por la existencia significa una lucha por el espacio...un pueblo superior que invade el territorio de su vecino más débil y salvaje lo despoja de sus tierras, lo obliga a recluirse en espacios demasiado pequeños para subsistir, y sigue invadiendo y apoderándose incluso de sus magras posesiones, hasta que el débil pierde los últimos restos de su dominio y es literalmente expulsado de la tierra (cit. en Bauman, 2006: 198).

\subsection{La plasticidad del reduccionismo: el enfrentamiento entre el poder naval y poder terrestre}

La historia de la humanidad puede plantearse como un enfrentamiento entre el poder naval y el terrestre, entre la talasocracia y lo telúrico. Es ciertamente reduccionista, simplificadora y nuevamente falaz, pero puede hacerse y se entiende bien; resulta sumamente sugestiva. Así, las guerras médicas que enfrentaron a la talasocracia griega con el poder continental representado el imperio persa, supuso la victoria del poder naval. En una de ellas el oráculo Fortificate con murallas de madera, que en su día pronunciara la sibila trajo, de la mano de Temistocles y su libre interpretación, la victoria de los griegos en Salamina. Las guerras del Peloponeso dieron finalmente la victoria a la continental Esparta frente a los marítimos atenienses. Los fenicios por su parte contrataban piratas para escoltar a su flota mercante. El poder continental de Roma venció en las tres guerras púnicas a la talasocracia cartaginesa, una vez que hubo aniquilado su poder marítimo. No obstante quedaron grupos piráticos en el Mare Nostrum que solo pudieron ser aniquilados por Cneo Pompeyo atacando sus bases en tierra, lo cual es una forma muy normal de resolver los problemas en la mar. Una Bizancio debilitada por las cruzadas, también se vio aniquilada por el poder continental turco. La visión continental de España se vio a su vez derrotada por la concepción marítima de la monarquía británica, al menos según la historiografía anglosajona. No obstante, el sistema de convoyes fue un avance, que se reeditaría en la Segunda Guerra Mundial, por su éxito que limitó solo al $1 \%$ las pérdidas debidas al ataque de corsarios y piratas.

En cualquier caso, el resultado de la Primera y Segunda Guerra Mundial se decidió en los mares y la Guerra Fría enfrento a una potencia continental con una forjada sobre los esquemas de Mahan con el resultado conocido por todos. 


\subsection{La Geopolítica binaria de Mahan}

Mahan es el apóstol del poder naval al que eleva por encima de cualquier otra forma de acción militar. El poder naval, a su juicio, prevalece sobre el poder terrestre. Pretende que las marinas actúen de forma totalmente autónoma, identificando el dominio del mar con la victoria. Para ello propone la concentración de fuerzas, una actuación ofensiva, en la que todas las operaciones principales se encuentran ligadas entre sí y, en la línea de Clausewitz - aunque él es más discípulo de Jomini trasladado al término naval, el intérprete del momento de moda del siglo hasta 1870 en que lo sustituyera el prusiano-, la batalla decisiva frente al enemigo. Todo ello de la mano de una marina en la que la pieza clave eran los grandes acorazados.

$\mathrm{Su}$ trabajo se centra en la importancia histórica del control del mar y el rol que éste ha desempeñado en la historia de la humanidad, siendo la conclusión general que puede extraerse de esta, la necesidad de mantener las líneas de comunicación para uso propio y negárselas al enemigo que así ve estrangulada su economía desde el mar. Para ello ofrece un modelo a seguir, el británico, cuya historia se toma como referencia y ejemplo. Describe el poder marítimo de una nación como la capacidad para crear, desarrollar, explotar y defender el mar en su beneficio. Se compone de intereses marítimos y el poder naval para su defensa.

El poder marítimo se construye sobre un comercio exterior próspero, al que sigue una poderosa marina mercante defendida por una marina de guerra dimensionada de modo acorde; ésta cuenta con bases marítimas para sus navíos y, cómo no en un contexto imperialista, de unas colonias que supongan el aporte de materias primas.

Su pensamiento en relación con Estados Unidos se centra sobre tres conceptos geopolíticos. En primer término, el país habría de dotarse de una flota y controlar el entorno cercano; en segundo lugar, debería tener presencia naval en aquellas aguas más alejadas de territorio norteamericano, $\mathrm{y}$, finalmente, dominar los mercados y los puertos de apoyo necesarios para, cerrando el círculo, reforzar por el mar el poder nacional y su economía. Es esencial, pues, en su propuesta la creación escalonada de una flota capaz de actuar a nivel global.

Al objeto de conseguir ese efecto, y en virtud del principio de concentración, es imprescindible la capacidad de conexión entre los dos mares del país continente. Por eso apoyó la construcción del Canal de Panamá; y no sólo eso sino que también, y como elemento clave de su propuesta geopolítica, propugnaba un férreo control sobre el mar Caribe - que calificaba como una suerte de Mediterráneo, el Mediterráneo americano; hablaba también de otro Mediterráneo que ubicaba en Asia-Pacífico-, el Golfo de México y el control de Hawaii.

Otra relevante aportación de Mahan es el concepto de "insularidad", que no sólo alcanza a países como Japón y Gran Bretaña, sino que a ellos se suman otros Estados que, ocupando una posición continental y sin tener vecinos continentales como 
rivales potenciales, también debían ser catalogados como "estratégicamente insulares". Tal es el caso de los Estados Unidos.

Esta insularidad si bien es cierto que en principio situaría a los países en la periferia geopolítica, en la práctica les confiere libertad de acción para escoger momento y lugar para actuar mientras queda a resguardo de los conflictos continentales. Sus ideas serían desarrolladas por pensadores actuales como Zbigniew Kazimierz Brzezinski o Paul Kennedy. En Eurasia, "el gran tablero" de Brzezinski (1998), la posición insular de los Estados Unidos, alejada del escenario pero vinculada con él por el mar, ofrece una gran ventaja. Los Estados Unidos son la única gran potencia que puede elegir si actúa o no en determinada área del continente clave, precisamente porque está fuera y tiene asegurado el acceso a través de los océanos. De esta manera, en esa posición de ventaja, lo único necesario es mantener el pluralismo geoestratégico dentro del tablero, evitando que alguien sea capaz de dominarlo todo. Evitar la hegemonía en Eurasia de una potencia y mantener el control del mar son suficientes para garantizar una posición geopolítica privilegiada que concede a los Estados Unidos por siempre la condición de nación indispensable. Este enfoque geopolítico es una reedición a nivel global de la política europea del Reino Unido desde el siglo XVII.

Evidentemente tanto Paul Kennedy como Zbigniew Brzezinski están de acuerdo en que la clave del Poder Marítimo deriva de su relación con la superficie de la Tierra. Tal poder es de naturaleza instrumental, es una función y como tal encarna una paradoja: El Poder Marítimo se busca y se ejerce para influir en los acontecimientos en tierra. En palabras del Almirante Dudley Knox: "La más alta confirmación del estratega naval es el calado de su comprensión [...] de la realidad de que [...] todo esfuerzo en la mar debe dirigirse a obtener un efecto en tierra" (cit. en VV AA, 2011: 27). Se trata de que los buques no hagan solamente, parafraseando a Machado, "estelas en la mar".

Los grandes teóricos del poder marítimo son Mahan y Corbett, autor de la obra Some Principles of Maritime Strategy, a los que se une el español Sánchez de Toca, autor del primer plan naval español del siglo XX. Mahan, en la línea de Clausewitz, es partidario de la supremacía total, el dominio, y de la aniquilación del enemigo en la batalla decisiva. Corbett, postula una aproximación indirecta al modo propuesto por Lidell Hart y defiende el control local materializado en el control de las líneas sobre los accesos y las comunicaciones. La Segunda Guerra Mundial tuvo en el escenario del Pacífico un enfrentamiento entre los criterios de Mahan y Corbett. Mahan se encontraba representado por la política estratégica del Japón que buscaba la batalla decisiva y Corbett por los EE UU que atacaba las líneas de comunicaciones. Curiosa situación que no deja de relevar la amplia influencia de la obra de Mahan más allá de sus fronteras y especialmente en el Imperio japonés y en el alemán del Kaiser. 


\section{Referencia y vigencia del pensamiento de Mahan}

El punto de partida del análisis geopolítico es que el pensamiento geopolítico se construye desde una atalaya, desde un observatorio. Si se modifican las coordenadas, si se varía el observatorio, si se cambia de posición o el momento, si cambia el tiempo o el espacio, el producto geopolítico será otro. Por eso es muy importante empezar descubriendo quién dice qué. Lo primero es identificar al observador y su posición. El punto de partida es descubrir la posición del observador, levantar sus coordenadas, sabiendo quién es y dónde está se entenderá mejor lo que ve, lo que cuenta y por qué lo cuenta. No hay una geopolítica sino muchas.

Mahan mira al mar y a su país y concluye con una política que liga ambos términos de referencia. Si, por un lado, el producto es toda una política exterior, por el otro, obtiene una política naval. Y es que a finales del siglo XIX Estados Unidos consolidó su poder territorial y se hizo consciente de su dimensión costera, descubrió el mar, lo que motivó el que pasase de contar con una marina de tamaño reducido, a convertirse en pocos años y apoyándose en su poderosa industria en una de las marinas más potentes del mundo; la marina norteamericana era la tercera del mundo en 1900 y en 1908 se convirtió en la segunda.

Es esta una época en que, tras la revolución de los transportes, las marinas eran el único expositor allende sus fronteras, de la capacidad industrial y militar de sus respectivas naciones, prácticamente lo único visible de ellas. Una de las actividades más importantes llevadas a cabo por los buques es lo que en clave naval se llama "enseñar la bandera".

A pesar de que el modelo de Mahan tras un siglo de su formulación se ha visto ampliamente superado, y que el mundo ha mutado considerablemente, sus propuestas siguen siendo un clásico al que se recurre con mucha frecuencia. La explicación para la pervivencia de sus ideas es doble. Por una parte, la política que él preconizaba se materializó, y aun es más, el autor contribuyó activamente a su diseño ocupando puestos por encima de su estatus profesional; gozó de una gran capacidad de influencia y acceso directo a los líderes políticos del país ante los que actuaba como una suerte de apóstol del poder naval. Por otra, su discurso, bien articulado y construido, por el lado naval contribuyó a la elaboración del concepto de "Sea Power", una idea talasocrática que tendría su contestación, nolens volens, en el discurso telúrico de Mackinder (2010 [1904]), discurso perfectamente sólido y válido, únicamente que construido desde otra atalaya.

$\mathrm{Su}$ obra encarna un poderoso lenguaje de justificación que convierte a Mahan aun hoy en una referencia necesaria, en un lugar común por el que es preceptivo transitar en cualquier alegato para justificar la necesidad y el papel de todas las marinas del mundo. Estas no dudan en sacar a Mahan de su tumba - hasta la española, tan maltratada por él- para justificarse. De hecho, la obra de Mahan es clásica, algo de la que pueden hablar muchos, aun sin haberla leído. 


\section{Conclusión}

Durante una conferencia en el Naval War College en 1892 Mahan afirmaba: "Caballeros, todo el mundo sabe que estamos creando una nueva Marina... Bien ¿Y cuando la tengamos, qué vamos a hacer con ella?" (cit. en Crowl, 1992: 491). Esta consideración nos demuestra que en este caso, como en otros muchos, es la ecuación de poder quien determina la geoestrategia. Es decir son los medios y recursos los que deciden qué es lo que se hace en función de lo que se puede hacer, de alguna forma la estrategia de la acción viene después de la estrategia genética de la fuerza o del poder. Si tenemos un martillo muchos problemas tendrán forma de clavo. Esta realidad es la idea clave del famoso ensayo de Robert Kagan titulado Poder y debilidad: Europa y Estados Unidos en el nuevo orden mundial. Allí Kagan nos dice que la diferencia clave entre las dos orillas del Atlántico no tiene tanto que ver con una brecha de filosofía política o cultura estratégica sino fundamentalmente con la distancia entre las capacidades militares de los Estados Unidos y de Europa. No es la estrategia la que diseña los medios son los medios los que diseñan la estrategia:

Las diferencias psicológicas entre el poder y la debilidad son fáciles de entender: a un hombre sin más armas que un cuchillo seguramente le parecerá que un oso que merodea por el bosque es un peligro tolerable, puesto que la alternativa, darle muerte armado sólo con un cuchillo, entraña más riesgos que tumbarse inmóvil y confiar en que el oso no ataque. El mismo hombre armado con un rifle, sin embargo, probablemente hará un cálculo diferente de qué constituye un riesgo tolerable (Kagan, 2003: $50)$.

Lo que Kagan plantea en términos de peligro se puede también plantear en términos de oportunidad, como sucedió en la guerra contra España en 1898. Pero como siempre las cuestiones preliminares son las más importantes. Utilizando el ejemplo de Kagan, lo primero que habría que poner sobre la mesa es el interés de su hombre por el bosque donde viven las fieras. Si el oso vive en el bosque solo es una amenaza para el hombre que lo descubre como un espacio de acción y negocio. Mahan precisamente lo que hizo fue eso, descubrir el bosque americano en el mar y construir un discurso para que sus compatriotas identificasen el nuevo tablero de juego, que de repente surgía en medio de los árboles en el océano.

Este pequeño paso para el hombre pero grande para la humanidad cambio la panorámica del mundo y su papel en él de los norteamericanos. La nueva visión y la nueva frontera abrían una brecha en la conceptualización americana de sus relaciones con el resto del planeta. El aislacionismo originario en el mito de la puritana república americana podía mutar y dar un salto al agua, para hacer del intervencionismo una necesidad de seguridad del faro que brilla. 
En el primer capítulo de su libro Diplomacia, Henry Kissinger (1994) dice que los Estados Unidos a lo largo de toda su historia ha mantenido dos actitudes contradictorias en su política exterior. Estamos completamente de acuerdo con este juicio. La contradicción y la esquizofrenia son algo particularmente propio de la política exterior norteamericana y probablemente de la política exterior de cualquier democracia. Las dos actitudes contradictorias, las dos categorías, definen un esquema clásico y sencillo muy utilizado para aproximarse a la política americana. Por un lado la mejor forma en que los Estados Unidos sirven a sus valores es perfeccionando la democracia del propio país, actuando como faro para el resto de la humanidad; por otro, los valores la nación norteamericana imponen la obligación de defenderlos en todo el mundo, como si de una cruzada se tratase. Intereses y valores, con todas las contradicciones inherentes a la mezcla de conceptos distintos, con una praxis pendular que oscila entre unos y otros.

Aceptar este modelo, con todo lo que puede tener de esclarecedor y al tiempo de simplificador, puede valer sólo si entendemos que los Estados Unidos empezaron a tener historia diplomática a partir de la presidencia de Theodore Roosevelt, algo a lo que Mahan sin duda contribuyó notablemente. Consideración al parecer compartida por Kissinger (1994), que comienza su Diplomacia descubriéndonos el giro de la presidencia del amigo del garrote a la del presidente Wooddrow Wilson. Es decir, en los Estados Unidos estas dos escuelas de pensamiento, la de los Estados Unidos como faro y la de los Estados Unidos como cruzado, existen en pugna desde la guerra hispano norteamericana de 1898.

La segunda escuela, la de los cruzados de la democracia, tiene mucho que ver con el descubrimiento del mar, la armada y los marines. Pues bien, la primera piedra de esta escuela de luchadores universales por la libertad y el comercio la puso Mahan, aunque posiblemente sin adivinar su trascendencia. Mahan subió a América a una nueva atalaya desde la que se veía todo el mundo como una unidad cerrada y que se podía representar colocando en el centro a los Estados Unidos de América abrazando al resto con los océanos.

Las ideas, ilusiones, mitos y palabras de un relato son el cimiento sobre el que se construye la visión del orden del mundo. Este relato es imprescindible para movilizar la proyección creadora de un nuevo poder nacional en el exterior. Escribir este relato es trabajo de unos pocos y las consecuencias, cuando la nación lo hace suyo, afectan a varias generaciones.

Unos escriben el guion, otros lo representan, otros pagan la entrada y aplauden al final, o no. Cada uno tiene su papel pero cuando al pasar el tiempo la obra sigue representándose acaba convirtiéndose en un clásico. A los promotores del espectáculo, aunque les gusta la novedad en la puesta en escena, los clásicos siempre les apasionan, porque un clásico siempre sugiere una reinterpretación del destino de una vida. Los clásicos son eternos para una civilización y Mahan parece haberse convertido en uno de ellos. Antes que cualquier ecuación de poder o debilidad hay un relato que la explica. 


\section{Bibliografía}

Agnew, John A. (2005) Geopolítica: una revisión de la política mundial. Madrid: Trama Editorial.

Bauman, Zygmunt (2006) Modernidad líquida. Buenos Aires: Fondo de Cultura Económica.

Brodie, Bernard (1978) Guerra y política. México. Fondo de Cultura Económica.

Brzezinski, Zbigniew (1998) El tablero mundial. La supremacía estadounidense y sus imperativos geoestratégicos. Barcelona: Paidós [ed. original en inglés The Grand Chessboard. American Primacy and its Geostrategic Imperatives. Nueva York: Basic Books, 1997].

Cairo, Heriberto (2005) "Prólogo. Re-pensando la Geopolítica: la renovación de la disciplina y las aportaciones de John A. Agnew", en J. A. Agnew: Geopolítica: una revisión de la política mundial. Madrid: Trama Editorial, ix-xvi.

Cervera Pery, José (2000) La Guerra Naval del 98. Madrid: Editorial San Martín Crowl, Philip A. (1992) “Alfred Tayer Mahan. El Historiador Naval”, en P. Paret (ed.) Creadores de la Estrategia Moderna. Madrid: Ministerio de Defensa, 461494.

Fanconi, Paloma (1995) Quevedo: De la mundana falsedad y las vanidades de los hombres. Madrid: Ediciones Temas de Hoy.

Jiménez, Alfredo (2001) "La historia como fabricación del Pasado: la frontera del Oeste". Anuario de Estudios Americanos, vol. LVIII, núm. 2.

Kagan, Robert (2003) Poder y debilidad: Europa y Estados Unidos en el nuevo orden mundial. Madrid: Taurus.

Kershaw, Ian (2010) Hitler. Barcelona: Ediciones Península.

Kissinger, Henry (1994) Diplomacia. México: Fondo de Cultura Económica.

Kjellen, Rudolf (1916) Staten som Lifsform. Estocolmo: Hugo Gebers Förlag.

Mackinder, Halford J. (2010) "El pivote geográfico de la historia". Geopolítica(s). Revista de estudios sobre espacio y poder, 1(2), 301-319 [edición original "The geographical pivot of history". The Geographical Journal, 23(4), 1904, 421437].

Mahan, Alfred T. (1883) The Gulf and Inland Waters. Nueva York: Charles Scribner's sons. [URL: <http://www.gutenberg.org/files/21562/21562-h/21562h.htm>. Consultado el 12 de Diciembre de 2013].

Mahan, Alfred T. (1890) The Influence of Sea Power Upon History: 1660-1783. Boston: Little, Brown and Co. [trad. al castellano por los Tenientes de Navío Juan Cervera y Jácome y Gerardo Sobrini y Argullos: La influencia del poder naval en la historia 1660-1783. Ferrol: Imprenta de El Correo Gallego,1901 (Copia autorizada por el Estado Mayor de la Armada, "Declarada de texto para las conferencias y lecturas de los Guardias Marinas, según el Reglamento actualmente vigente, Madrid, Junio de 1974)]. 
Paret, Peter (1992) Creadores de la Estrategia Moderna. Desde Maquiavelo a la Era Nuclear. Madrid: Ministerio de Defensa.

Pérez Tudela Baez, José María et al. (1992) Historia del mundo contemporáneo. Madrid: Editorial Edinumen.

Tucídides (1990) Historia de la guerra del Peloponeso. Madrid: Gredos.

Turner, Frederick Jackson (1893) The significance of the frontier in American History. Indianapolis: Bobbs-Merril.

VV AA (2011) Conferencia "El Poder naval". Madrid: Escuela Superior de las Fuerzas Armadas.

Zorrilla, José (2013) "Bienvenidos a la Edad de oro del conflicto". Diario "El Confidencial", 13 de Diciembre [URL: $<$ http://www.elconfidencial.com/mundo/2013-12-30/bienvenidos-a-la-edad-deoro-del-conflicto_70764/>. Consultado el 13 de Diciembre de 2013]. 